\title{
Analysis of Bidirectional Ballot Sequences and Random Walks Ending in Their Maximum
}

\author{
Benjamin Hackl ${ }^{1 *}$, Clemens Heuberger ${ }^{1 *}$, Helmut Prodinger ${ }^{2 \dagger}$, and Stephan Wagner ${ }^{2 \dagger}$ \\ ${ }^{1}$ Institut für Mathematik, Alpen-Adria-Universität Klagenfurt, Universitätsstraße 65-67, 9020 \\ Klagenfurt, Austria \\ \{benjamin.hackl, clemens.heuberger\}@aau.at \\ ${ }^{2}$ Department of Mathematical Sciences, Stellenbosch University, 7602 Stellenbosch, South \\ Africa \\ \{hproding, swagner\}@sun.ac.za
}

Received March 31, 2015

Mathematics Subject Classification: 05A16; 05A15, 05A10, 60C05

\begin{abstract}
Consider non-negative lattice paths ending at their maximum height, which will be called admissible paths. We show that the probability for a lattice path to be admissible is related to the Chebyshev polynomials of the first or second kind, depending on whether the lattice path is defined with a reflective barrier or not. Parameters like the number of admissible paths with given length or the expected height are analyzed asymptotically. Additionally, we use a bijection between admissible random walks and special binary sequences to prove a recent conjecture by Zhao on ballot sequences.
\end{abstract}

Keywords: lattice path, culminating path, ballot sequence, asymptotic expansion, Chebyshev polynomial

\section{Introduction}

Lattice paths as well as their stochastic incarnation — random walks — are interesting and classical objects of study. Several authors have investigated a variety of parameters related to lattice paths. For example, Banderier and Flajolet gave an asymptotic analysis of the number of special lattice paths with fixed length in [2]. De Brujin, Knuth, and Rice [4] analyzed the expected height of certain lattice paths, and Panny and Prodinger [14] determined the asymptotic behavior of such paths with respect to several notions of height.

The particular class of lattice paths we want to analyze in this paper is defined as follows.

\footnotetext{
${ }^{*}$ B. Hackl and C. Heuberger are supported by the Austrian Science Fund (FWF): P 24644-N26.

${ }^{\dagger} \mathrm{H}$. Prodinger is supported by an incentive grant of the National Research Foundation of South Africa.

${ }^{\ddagger}$ S. Wagner is supported by the National Research Foundation of South Africa, grant number 96236 .
} 
Definition 1.1. (Admissible random walks and lattice paths) Let $\left(S_{k}\right)_{0 \leq k \leq n}$ be a simple symmetric random walk on $\mathbb{N}_{0}$ or $\mathbb{Z}$ of length $n$ starting at 0 . That is, we have $\mathbb{P}\left(S_{0}=0\right)=1$ as well as

$$
\begin{aligned}
& \mathbb{P}\left(S_{k}=j-1 \mid S_{k-1}=j\right)=\mathbb{P}\left(S_{k}=j+1 \mid S_{k-1}=j\right)=\frac{1}{2}, \quad \text { for } j \geq 1, \\
& \mathbb{P}\left(S_{k}=1 \mid S_{k-1}=0\right)=1,
\end{aligned}
$$

for random walks defined on $\mathbb{N}_{0}$, and

$$
\mathbb{P}\left(S_{k}=j-1 \mid S_{k-1}=j\right)=\mathbb{P}\left(S_{k}=j+1 \mid S_{k-1}=j\right)=\frac{1}{2}, \quad \text { for } j \in \mathbb{Z},
$$

for random walks on $\mathbb{Z}$. Then $\left(S_{k}\right)_{0 \leq k \leq n}$ is said to be admissible of height $h$, if the random walk stays within the interval $[0, h]$ and ends in $h$, i.e., $S_{k} \in[0, h]$ for all $0 \leq k \leq n$ and $S_{n}=h$. It is called admissible, if it is admissible of height $h$ for some $h \in \mathbb{N}$.

The probability that a random walk of length $n$ is admissible of height $h$ is written as $p_{n}^{(h)}$ and $q_{n}^{(h)}$ for random walks on $\mathbb{N}_{0}$ and $\mathbb{Z}$, respectively. Furthermore, the probabilities that a random walk is admissible at all are defined as $p_{n}:=\sum_{h \geq 0} p_{n}^{(h)}$ and $q_{n}:=\sum_{h \geq 0} q_{n}^{(h)}$, respectively.

Finally, an admissible lattice path is a sequence of integers realizing an admissible random walk.

In a nutshell, this means that an admissible random walk is a non-negative random walk ending in its maximum. The definition is also visualized in Figure 1, where all admissible lattice paths of length 5 are depicted. There are three admissible lattice paths of height 3 , and one of height 1 and 5, respectively. Note that when considering random walks on $\mathbb{Z}$, every lattice path has the same probability $2^{-n}$. Admissible random walks on $\mathbb{Z}$ are enumerated by sequence $A 167510$ in [11].

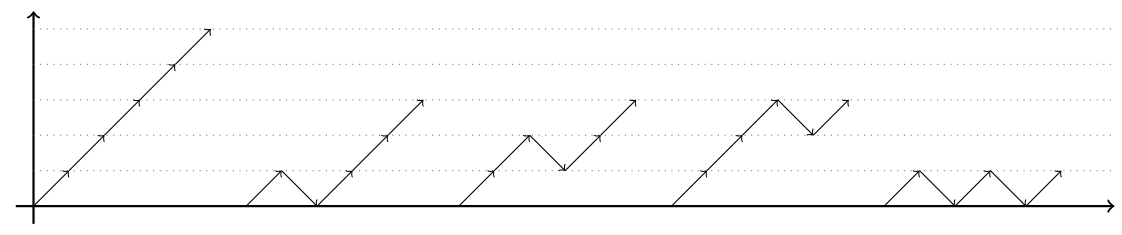

Figure 1: Admissible lattice paths of length 5.

However, in the case of random walks on $\mathbb{N}_{0}$, the probability depends on the number of visits to 0: if there are $v$ such visits (including the initial state), then the path occurs with probability $2^{-n+v}$. Note that by "folding down" (i.e., reflecting about the $x$-axis) some sections between consecutive visits to 0 , or the section between the last visit and the end, $2^{v}$ lattice paths on $\mathbb{Z}$ can be formed, where the random walk is never farther away from the start than at the end. We will call such lattice paths extremal lattice paths - and by construction, the number of extremal lattice paths of 


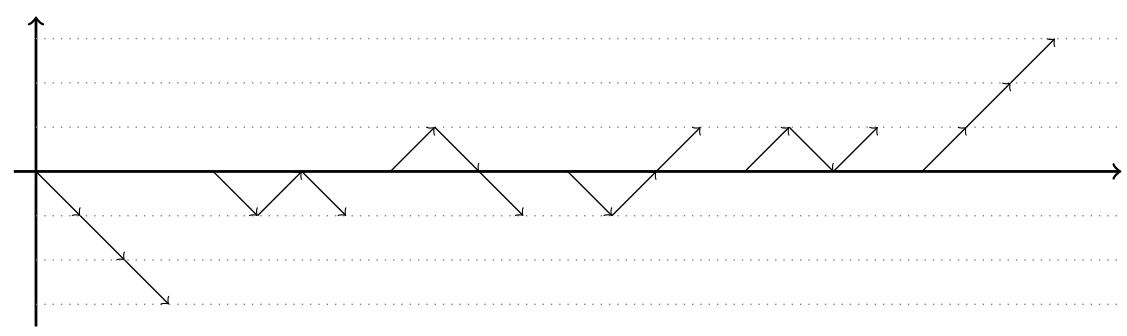

Figure 2: Extremal lattice paths of length 3.

length $n$ is given by $p_{n} 2^{n}$. To illustrate this idea of extremal lattice paths, all paths of this form of length 3 are given in Figure 2.

One of our motivations for investigating admissible random walks originates from a conjecture in [16]. There, Zhao introduced the notion of a bidirectional ballot sequence:

Definition 1.2. ([16, Definition 3.1]) A 0-1 sequence is called a bidirectional ballot sequence if every prefix and suffix contains strictly more 1's than 0's. The number of bidirectional ballot sequences of length $n$ is denoted by $B_{n}$.

Bidirectional ballot sequences are strongly related to admissible random walks on $\mathbb{Z}$. In fact, every bidirectional ballot sequence of length $n+2$ bijectively corresponds to an admissible random walk of length $n$ on $\mathbb{Z}$ : given an admissible random walk, every up-step corresponds to a 1 , and down-steps correspond to 0 . Adding a 1 both at the beginning and at the end of the constructed string gives a bidirectional ballot sequence of length $n+2$.

Therefore, bidirectional ballot walks may also be seen as lattice paths with unique minimum and maximum.

While we restrict ourselves to simple lattice paths (i.e., the path has steps \pm 1 ), Bousquet-Mélou and Ponty introduce a more general class of so-called culminating paths in [3]. Akin to bidirectional ballot walks, culminating paths are lattice paths with unique minimum and maximum - however, the lattice path has steps $a$ and $-b$ for fixed $a, b>0$. They show that the behavior of these paths strongly depends on the drift $a-b$. In particular, for $a=b=1$ (i.e., for bidirectional ballot walks) they determine the main term of the asymptotic expansion of $B_{n}$ (cf. [3, Proposition 4.1]).

In [16], Zhao also shows that $B_{n}=\Theta\left(2^{n} / n\right)$, states (without detailed proof) that $B_{n} \sim 2^{n} /(4 n)$ and conjectures that

$$
\frac{B_{n}}{2^{n}}=\frac{1}{4 n}+\frac{1}{6 n^{2}}+O\left(\frac{1}{n^{3}}\right)
$$

In this paper, we want to give a detailed analysis of the asymptotic behavior of admissible random walks. By exploiting the bijection between admissible random walks and bidirectional ballot sequences, we also prove a stronger version of Zhao's conjecture.

In order to do so, we use a connection between Chebyshev polynomials and the probabilities $p_{n}^{(h)}$ and $q_{n}^{(h)}$ (cf. Propositions 2.1 and 2.2, respectively), which we ex- 
plore in detail in Section 2. This allows us to determine explicit representations of the probabilities $p_{n}$ and $q_{n}$, which are given in Theorem 2.5 . The analysis of the asymptotic behavior of admissible random walks of given length shall focus in particular on the height of these random walks. In this context, we define random variables $H_{n}$ and $\widetilde{H}_{n}$ by

$$
\mathbb{P}\left(H_{n}=h\right):=\frac{p_{n}^{(h)}}{p_{n}}, \quad \mathbb{P}\left(\widetilde{H}_{n}=h\right):=\frac{q_{n}^{(h)}}{q_{n}} .
$$

These random variables model the height of admissible random walks on $\mathbb{N}_{0}$ and $\mathbb{Z}$, respectively. Besides an asymptotic expansion for $p_{n}$ and $q_{n}$, we are also interested in the behavior of the expected height and its variance. The asymptotic analysis of these expressions, which is based on an approach featuring the Mellin transform, is carried out in Sections 3 and 4, and the results are given in Theorems 3.5 and 4.2, respectively. Finally, Zhao's conjecture is proved in Corollary 4.5.

\section{Chebyshev Polynomials and Random Walks}

We denote the Chebyshev polynomials of the first and second kind by $T_{h}$ and $U_{h}$, respectively, i.e.,

$$
\begin{array}{ll}
T_{h+1}(x)=2 x T_{h}(x)-T_{h-1}(x), & \text { for } h \geq 1, T_{0}(x)=1, T_{1}(x)=x, \\
U_{h+1}(x)=2 x U_{h}(x)-U_{h-1}(x), & \text { for } h \geq 1, U_{0}(x)=1, U_{1}(x)=2 x .
\end{array}
$$

In the following propositions, we show that these polynomials occur when analyzing admissible random walks. As usual, the notation $\left[z^{n}\right] f(z)$ denotes the coefficient of $z^{n}$ in the series expansion of $f(z)$.

Proposition 2.1. The probability that a simple symmetric random walk $\left(S_{k}\right)_{0 \leq k \leq n}$ of length $n$ on $\mathbb{Z}$ is admissible of height $h$ is

$$
q_{n}^{(h)}=\mathbb{P}\left(0 \leq S_{0}, S_{1}, \ldots, S_{n} \leq h \text { and } S_{n}=h\right)=2\left[z^{n+1}\right] \frac{1}{U_{h+1}(1 / z)},
$$

for $h \geq 0$ and $n \geq 0$.

Proof. We consider the $(h+1) \times(h+1)$ transfer matrix

$$
M_{h}=\left(\begin{array}{cccccc}
0 & \frac{1}{2} & 0 & \ldots & \ldots & 0 \\
\frac{1}{2} & 0 & \frac{1}{2} & \ldots & \ldots & 0 \\
0 & \frac{1}{2} & 0 & \ddots & & 0 \\
\vdots & \vdots & \ddots & \ddots & \ddots & \vdots \\
\vdots & \vdots & & \ddots & \ddots & \frac{1}{2} \\
0 & 0 & 0 & \ldots & \frac{1}{2} & 0
\end{array}\right)
$$

which has the following simple yet useful property: if $w_{n, k}^{(h)}$ is the probability that $0 \leq S_{0}, S_{1}, \ldots, S_{n} \leq h$ and $S_{n}=k$, then the following recursion for the vectors $w_{n}^{(h)}=$ 
$\left(w_{n, 0}^{(h)}, w_{n, 1}^{(h)}, \ldots, w_{n, h}^{(h)}\right)$ holds:

$$
w_{n}^{(h)} \cdot M_{h}=w_{n+1}^{(h)},
$$

so $w_{n}^{(h)}=w_{0}^{(h)} \cdot M_{h}^{n}$. The initial vector is $w_{0}^{(h)}=e_{0}=(1,0, \ldots, 0)$. Since we also want that $S_{n}=h$, we multiply by the vector $e_{h}=(0, \ldots, 0,1)^{\top}$ at the end to extract only the last entry $w_{n, h}^{(h)}$. This yields the generating function

$$
\sum_{n \geq 0} q_{n}^{(h)} z^{n}=\sum_{n \geq 0} e_{0} M_{h}^{n} e_{h} z^{n}=e_{0}\left(I-z M_{h}\right)^{-1} e_{h} .
$$

Cramer's rule yields

$$
\sum_{n \geq 0} q_{n}^{(h)} z^{n}=\frac{z^{h} 2^{-h}}{\operatorname{det}\left(I-z M_{h}\right)} .
$$

The determinant of $I-z M_{h}$ can be computed recursively in $h$ by means of row expansion, see (for instance) [1, p. 97]:

$$
\operatorname{det}\left(I-z M_{h+2}\right)=\operatorname{det}\left(I-z M_{h+1}\right)-\frac{z^{2}}{4} \operatorname{det}\left(I-z M_{h}\right) .
$$

Comparing this with the recursion for the Chebyshev polynomials and checking the initial values, we find that $\frac{2^{h+1} \operatorname{det}\left(I-z M_{h}\right)}{z^{h+1}}=U_{h+1}(1 / z)$. Therefore, we obtain

$$
\sum_{n \geq 0} q_{n}^{(h)} z^{n}=\frac{2}{z U_{h+1}(1 / z)}
$$

from which (2.1) follows by extracting the coefficient of $z^{n}$.

An analogous statement holds for admissible random walks on $\mathbb{N}_{0}$ with the sole difference that in this case, the Chebyshev polynomials of the first kind occur.

Proposition 2.2. The probability that a random walk $\left(S_{k}\right)_{0 \leq k \leq n}$ of length $n$ on $\mathbb{N}_{0}$ is admissible of height $h$ is given by

$$
p_{n}^{(h)}=\mathbb{P}\left(0 \leq S_{0}, S_{1}, \ldots, S_{n} \leq h \text { and } S_{n}=h\right)=2\left[z^{n+1}\right] \frac{1}{T_{h+1}(1 / z)},
$$

for $h \geq 0$ and $n \geq 1$.

Proof. For random walks with a reflective barrier at 0 , the $(h+1) \times(h+1)$ transfer matrix has the form

$$
\widetilde{M}_{h}=\left(\begin{array}{cccccc}
0 & 1 & 0 & \ldots & \cdots & 0 \\
\frac{1}{2} & 0 & \frac{1}{2} & \cdots & \cdots & 0 \\
0 & \frac{1}{2} & 0 & \ddots & & 0 \\
\vdots & \vdots & \ddots & \ddots & \ddots & \vdots \\
\vdots & \vdots & & \ddots & \ddots & \frac{1}{2} \\
0 & 0 & 0 & \ldots & \frac{1}{2} & 0
\end{array}\right)
$$


By the same approach involving Cramer's rule as in the proof of Proposition 2.1, we find the generating function

$$
\sum_{n \geq 0} p_{n}^{(h)} z^{n}=e_{0}\left(I-z \widetilde{M}_{h}\right)^{-1} e_{h}=\frac{z^{h} 2^{1-h}}{\operatorname{det}\left(I-z \widetilde{M}_{h}\right)},
$$

where we have the recursion

$$
\operatorname{det}\left(I-z \widetilde{M}_{h+2}\right)=\operatorname{det}\left(I-z \widetilde{M}_{h+1}\right)-\frac{z^{2}}{4} \operatorname{det}\left(I-z \widetilde{M}_{h}\right),
$$

for the determinant of $I-z \widetilde{M}_{h}$. Finally, (2.2) follows from $\frac{2^{h-1} \operatorname{det}\left(I-z \widetilde{M}_{h-1}\right)}{z^{h}}=T_{h}(1 / z)$, which can be proved again by verifying that the same recursion holds for the Chebyshev- $T$ polynomials and that the initial values agree.

Remark 2.3. The coefficients of $\frac{1}{T_{h}(1 / z)}$ have also been studied in [9]. There, the case of fixed $h$ is investigated, whereas we mostly focus on the asymptotic behavior of $\sum_{h \geq 0} p_{n}^{(h)}$ for $n \rightarrow \infty$.

Using the results from Propositions 2.1 and 2.2, we may give explicit representations of the probabilities $p_{n}^{(h)}$ and $q_{n}^{(h)}$ by investigating the Chebyshev polynomials thoroughly.

Remark 2.4. (Iverson's notation) We use the Iversonian notation

$$
\llbracket \operatorname{expr} \rrbracket= \begin{cases}1, & \text { if expr is true } \\ 0, & \text { otherwise }\end{cases}
$$

popularized in [8, Chapter 2].

In the following theorem and throughout the rest of the paper, $m$ will denote a halfinteger, i.e., $m \in \frac{1}{2} \mathbb{N}=\left\{\frac{1}{2}, 1, \frac{3}{2}, 2, \ldots\right\}$. While this convention may seem unusual, it simplifies many of our formulae and is therefore convenient for calculations.

Theorem 2.5. With $\tau_{h, k}:=(h+1)(2 k+1) / 2$ and $v_{h, k}:=(h+2)(2 k+1) / 2$, we have

$$
\begin{aligned}
& p_{2 m-1}^{(h)}=\frac{4}{4^{m}} \sum_{k \geq 0}(-1)^{k} \frac{\tau_{h, k}}{m}\left(\begin{array}{c}
2 m \\
m-\tau_{h, k}
\end{array}\right) \cdot \llbracket h+1 \equiv 2 m \bmod 2 \rrbracket, \\
& q_{2 m-2}^{(h)}=\frac{4}{4^{m}} \sum_{k \geq 0} \frac{2 v_{h, k}^{2}-m}{(2 m-1) m}\left(\begin{array}{c}
2 m \\
m-v_{h, k}
\end{array}\right) \cdot \llbracket h \equiv 2 m \bmod 2 \rrbracket,
\end{aligned}
$$

for $h \geq 0$ and half-integers $m \in \frac{1}{2} \mathbb{N}$ with $m \geq 1$.

Proof. We begin with the analysis of $p_{n}^{(h)}$. The probabilities are related to the Chebyshev- $T$ polynomials by Proposition 2.2. It is a well-known fact (cf. [12, 22:3:3]) that these polynomials have the explicit representation

$$
T_{h}(x)=\frac{\left(x-\sqrt{x^{2}-1}\right)^{h}+\left(x+\sqrt{x^{2}-1}\right)^{h}}{2},
$$


which immediately yields

$$
\frac{1}{T_{h}(1 / z)}=z^{h} \frac{2}{\left(1-\sqrt{1-z^{2}}\right)^{h}+\left(1+\sqrt{1-z^{2}}\right)^{h}}=: z^{h} Y\left(z^{2}\right) .
$$

By applying Cauchy's integral formula, we obtain the coefficients of the factor $Y(t)$ encountered in (2.5). We choose a sufficiently small circle around 0 as the integration contour $\gamma$. Thus, we get

$$
\begin{aligned}
{\left[t^{n}\right] Y(t) } & =\left[t^{n}\right] \frac{2}{(1-\sqrt{1-t})^{h}+(1+\sqrt{1-t})^{h}} \\
& =\frac{1}{2 \pi i} \oint_{\gamma} \frac{2}{(1-\sqrt{1-t})^{h}+(1+\sqrt{1-t})^{h}} \cdot \frac{1}{t^{n+1}} d t .
\end{aligned}
$$

We want to simplify the expression $\sqrt{1-t}$ in this integral. This can be achieved by the substitution $t=\frac{4 u}{(1+u)^{2}}$, which gives us $d t=(1-u) \cdot \frac{4}{(1+u)^{3}} d u$ and $\sqrt{1-t}=\frac{1-u}{1+u}$. Also, the new integration contour is $\tilde{\gamma}$, which is still a contour that winds around the origin once. Then, again by Cauchy's integral formula, we obtain

$$
\begin{aligned}
{\left[t^{n}\right] Y(t) } & =\frac{1}{2 \pi i} \oint_{\tilde{\gamma}}(1-u) \frac{(1+u)^{2 n+h-1}}{2^{2 n+h-1}\left(1+u^{h}\right)} \cdot \frac{1}{u^{n+1}} d u \\
& =\left[u^{n}\right](1-u) \frac{(1+u)^{2 n+h-1}}{2^{2 n+h-1}\left(1+u^{h}\right)} .
\end{aligned}
$$

Expanding the factor $\frac{(1+u)^{2 n+h-1}}{1+u^{h}}$ into a series with the help of the geometric series and the binomial theorem yields

$$
\frac{(1+u)^{2 n+h-1}}{1+u^{h}}=\sum_{k \geq 0}(-1)^{k} u^{k h}(1+u)^{2 n+h-1}=\sum_{k \geq 0}(-1)^{k} u^{k h} \sum_{j=0}^{2 n+h-1}\left(\begin{array}{c}
2 n+h-1 \\
j
\end{array}\right) u^{j},
$$

and therefore,

$$
\left[u^{\ell}\right] \frac{(1+u)^{2 n+h-1}}{1+u^{h}}=\sum_{k \geq 0}(-1)^{k}\left(\begin{array}{c}
2 n+h-1 \\
\ell-h k
\end{array}\right) .
$$

This allows us to expand the expression encountered before, that is,

$$
\begin{aligned}
{\left[t^{n}\right] Y(t) } & =\left[u^{n}\right](1-u) \frac{(1+u)^{2 n+h-1}}{2^{2 n+h-1}\left(1+u^{h}\right)} \\
& =\frac{1}{2^{2 n+h-1}} \sum_{k \geq 0}(-1)^{k}\left[\left(\begin{array}{c}
2 n+h-1 \\
n-h k
\end{array}\right)-\left(\begin{array}{c}
2 n+h-1 \\
n-h k-1
\end{array}\right)\right] .
\end{aligned}
$$

Using the binomial identity

$$
\left(\begin{array}{c}
N-1 \\
\alpha
\end{array}\right)-\left(\begin{array}{c}
N-1 \\
\alpha-1
\end{array}\right)=\frac{N-2 \alpha}{N}\left(\begin{array}{l}
N \\
\alpha
\end{array}\right)
$$


the expression above can be simplified so that, together with (2.5), we find

$$
\frac{1}{T_{h}(1 / z)}=2 \sum_{n \geq 0}\left(\frac{z}{2}\right)^{2 n+h} \sum_{k \geq 0}(-1)^{k} \frac{2 h k+h}{2 n+h}\left(\begin{array}{c}
2 n+h \\
n-h k
\end{array}\right) .
$$

By plugging this into (2.2), we obtain

$$
\begin{aligned}
p_{n}^{(h)} & =2\left[z^{n+1}\right] \frac{1}{T_{h+1}(1 / z)} \\
& =4\left[z^{n+1}\right] \sum_{\ell \geq 0}\left(\frac{z}{2}\right)^{2 \ell+h+1} \sum_{k \geq 0}(-1)^{k} \frac{2(h+1) k+h+1}{2 \ell+h+1}\left(\begin{array}{c}
2 \ell+h+1 \\
\ell-(h+1) k
\end{array}\right) \\
& =\frac{1}{2^{h-1}}\left[z^{n-h}\right] \sum_{\ell \geq 0}\left(\frac{z}{2}\right)^{2 \ell} \sum_{k \geq 0}(-1)^{k} \frac{2(h+1) k+h+1}{2 \ell+h+1}\left(\begin{array}{c}
2 \ell+h+1 \\
\ell-(h+1) k
\end{array}\right) .
\end{aligned}
$$

Combinatorially, it is clear that $p_{n}^{(h)}=0$ for $n$ and $h$ of different parity, as only heights of the same parity as the length can be reached by a random walk starting at the origin. This can also be observed in the representation above. Assuming $n \equiv h \bmod 2$, we can write $n-h=2 \ell$ or equivalently $\frac{n-h}{2}=\ell$. This gives us

$$
\begin{aligned}
p_{n}^{(h)} & =\frac{1}{2^{n-1}} \sum_{k \geq 0}(-1)^{k} \frac{2(h+1) k+h+1}{n+1}\left(\begin{array}{c}
n+1 \\
\frac{n-h}{2}-(h+1) k
\end{array}\right) \\
& =\frac{1}{2^{n-1}} \sum_{k \geq 0}(-1)^{k} \frac{(h+1)(2 k+1)}{n+1}\left(\begin{array}{c}
n+1 \\
\frac{n+1}{2}-\frac{1}{2}(h+1)(2 k+1)
\end{array}\right) .
\end{aligned}
$$

Substituting $n=2 m-1$ with a half-integer $m \in \frac{1}{2} \mathbb{N}$ such that $h+1 \equiv 2 m \bmod 2$, and recalling that $\tau_{h, k}=(h+1)(2 k+1) / 2$, the representation in (2.3) is proved.

For the second part, we consider the explicit representation

$$
U_{h}(x)=\frac{\left(x+\sqrt{x^{2}-1}\right)^{h+1}-\left(x-\sqrt{x^{2}-1}\right)^{h+1}}{2 \sqrt{x^{2}-1}}
$$

of the Chebyshev- $U$ polynomials, which is equivalent to

$$
\frac{1}{U_{h}(1 / z)}=z^{h} \frac{2 \sqrt{1-z^{2}}}{\left(1+\sqrt{1-z^{2}}\right)^{h+1}-\left(1-\sqrt{1-z^{2}}\right)^{h+1}} .
$$

Formula (2.4) is now obtained in the same way as (2.3).

With explicit formulae for the probabilities $p_{n}^{(h)}$ and $q_{n}^{(h)}$, we can start to work towards the analysis of the asymptotic behavior of admissible random walks.

\section{Admissible Random Walks on $\mathbb{N}_{0}$}

In this section, we begin to develop the tools required for a precise analysis of the asymptotic behavior of admissible random walks on $\mathbb{N}_{0}$. 
Recalling the result of Theorem 2.5, we find that in the half-integer representation $p_{2 m-1}^{(h)}$, the shifted central binomial coefficient $\left(\begin{array}{c}2 m \\ m-\tau_{h, k}\end{array}\right)$ appears. Hence, for the purpose of obtaining an expansion for $p_{2 m-1}=\sum_{h \geq 0} p_{2 m-1}^{(h)}$, analyzing the asymptotics of binomial coefficients in the central region is necessary. In the following, we will work a lot with asymptotic expansions. The notation

$$
f(n) \sim \sum_{\ell=-L}^{\infty} a_{\ell} n^{-\ell}
$$

(as $n \rightarrow \infty)$ is understood to mean

$$
f(n)=\sum_{\ell=-L}^{R-1} a_{\ell} n^{-\ell}+O\left(n^{-R}\right)
$$

for all integers $R>-L$, even if the series does not converge. Likewise, an asymptotic expansion in two variables given by

$$
f(\alpha, n) \sim \sum_{\ell=-L}^{\infty} \sum_{j=0}^{J(\ell)} b_{\ell j} \frac{\alpha^{j}}{n^{\ell}}
$$

is to be understood as

$$
f(\alpha, n)=\sum_{\ell=-L}^{R-1} \sum_{j=0}^{J(\ell)} b_{\ell j} \frac{\alpha^{j}}{n^{\ell}}+O\left(\alpha^{J(R)} n^{-R}\right),
$$

for all $R>-L$.

Lemma 3.1. For $n \in \frac{1}{2} \mathbb{N}$ and $|\alpha| \leq n^{2 / 3}$ such that $n-\alpha \in \mathbb{N}$, we have

$$
\left(\begin{array}{c}
2 n \\
n-\alpha
\end{array}\right) \sim \frac{4^{n}}{\sqrt{n \pi}} \exp \left(-\frac{\alpha^{2}}{n}\right) \cdot S(\alpha, n),
$$

with $S(\alpha, n):=\sum_{\ell, j \geq 0} c_{\ell j} \frac{\alpha^{2 j}}{n^{\ell}}$ and

$$
\begin{aligned}
c_{\ell j}= & {\left[\alpha^{2 j} n^{-\ell}\right]\left(\sum_{r \geq 0} \frac{d_{r}}{(2 n)^{r}}\right)\left(\sum_{r \geq 0} \frac{(-1)^{r} d_{r}}{(n+\alpha)^{r}}\right)\left(\sum_{r \geq 0} \frac{(-1)^{r} d_{r}}{(n-\alpha)^{r}}\right) } \\
& \times\left(\sum_{r \geq 0}(-1)^{r}\left(\begin{array}{c}
-1 / 2 \\
r
\end{array}\right) \frac{\alpha^{2 r}}{n^{2 r}}\right)\left(\sum_{r \geq 0} \frac{1}{r !} \frac{\alpha^{4 r}}{n^{3 r}}\left[\sum_{t \geq 0} \frac{-1}{(t+2)(2 t+3)} \frac{\alpha^{2 t}}{n^{2 t}}\right]^{r}\right),
\end{aligned}
$$

where the coefficients $d_{r}$ come from the higher-order Stirling approximation of the factorial, cf. (3.2). Additionally, the estimate

$$
S(\alpha, n)=1+O\left(\frac{1+|\alpha|}{n}\right)
$$

holds for $|\alpha| \leq n^{2 / 3}$ and we know that $c_{00}=1$ as well as $c_{\ell j}=0$ if $j>\frac{2}{3} \ell$. 
If $|\alpha|>n^{2 / 3}$, the term

$$
\left(\begin{array}{c}
2 n \\
n-\alpha
\end{array}\right) / 4^{n}=O\left(\exp \left(-n^{1 / 3}\right)\right)
$$

decays faster than any power of $n$.

Proof. We begin by recalling the higher-order Stirling approximation (cf. [7, p. 760])

$$
n ! \sim \sqrt{2 \pi n}\left(\frac{n}{e}\right)^{n}\left(\sum_{j \geq 0} \frac{d_{j}}{n^{j}}\right) .
$$

An explicit representation of the coefficients $d_{j}$ can be found in [10]. From the logarithmic representation of the factorial (see [7, p. 766]), the expansion

$$
\frac{1}{n !} \sim \frac{1}{\sqrt{2 \pi n}}\left(\frac{e}{n}\right)^{n}\left(\sum_{j \geq 0} \frac{(-1)^{j} d_{j}}{n^{j}}\right)
$$

for the reciprocal factorial follows.

Let us assume $|\alpha| \leq n^{2 / 3}$. Then, by applying (3.2) and (3.3) to the shifted central binomial coefficient, we obtain

$$
\begin{aligned}
\left(\begin{array}{c}
2 n \\
n-\alpha
\end{array}\right)= & \frac{(2 n) !}{(n-\alpha) !(n+\alpha) !} \\
= & \frac{1}{\sqrt{n \pi}}\left(1-\frac{\alpha^{2}}{n^{2}}\right)^{-1 / 2} \frac{(2 n)^{2 n}}{(n+\alpha)^{n+\alpha}(n-\alpha)^{n-\alpha}} \\
& \times\left(\sum_{r \geq 0} \frac{d_{r}}{(2 n)^{r}}\right)\left(\sum_{r \geq 0} \frac{(-1)^{r} d_{r}}{(n+\alpha)^{r}}\right)\left(\sum_{r \geq 0} \frac{(-1)^{r} d_{r}}{(n-\alpha)^{r}}\right) .
\end{aligned}
$$

The factor $\left(1-\frac{\alpha^{2}}{n^{2}}\right)^{-1 / 2}$ can be expanded as a binomial series, resulting in

$$
\left(1-\frac{\alpha^{2}}{n^{2}}\right)^{-1 / 2}=\sum_{r \geq 0}(-1)^{r}\left(\begin{array}{c}
-1 / 2 \\
r
\end{array}\right) \frac{\alpha^{2 r}}{n^{2 r}} .
$$

The remaining factor is handled by means of the identity $n^{n}=\exp (n \log n)$, which leads to

$$
\begin{aligned}
& \frac{(2 n)^{2 n}}{(n+\alpha)^{n+\alpha}(n-\alpha)^{n-\alpha}} \\
= & \exp (2 n \log (2 n)-(n+\alpha) \log (n+\alpha)-(n-\alpha) \log (n-\alpha)) \\
= & \exp (2 n \log 2+2 n \log n-(n+\alpha)(\log n+\log (1+\alpha / n)) \\
& -(n-\alpha)(\log n+\log (1-\alpha / n))) \\
= & 4^{n} \exp (\alpha \log (1-\alpha / n)-\alpha \log (1+\alpha / n) \\
& -n \log (1-\alpha / n)-n \log (1+\alpha / n)) .
\end{aligned}
$$


By expanding the logarithm into a power series, we can simplify this expression to

$$
\begin{aligned}
& \frac{(2 n)^{2 n}}{(n+\alpha)^{n+\alpha}(n-\alpha)^{n-\alpha}} \\
& =4^{n} \exp \left(2\left[-\sum_{t \geq 0} \frac{1}{2 t+1} \frac{\alpha^{2 t+2}}{n^{2 t+1}}+\sum_{t \geq 0} \frac{1}{2 t+2} \frac{\alpha^{2 t+2}}{n^{2 t+1}}\right]\right) \\
& =4^{n} \exp \left(-\frac{\alpha^{2}}{n}\right) \exp \left(-\frac{\alpha^{4}}{n^{3}} \sum_{t \geq 0} \frac{1}{(t+2)(2 t+3)} \frac{\alpha^{2 t}}{n^{2 t}}\right) \\
& =4^{n} \exp \left(-\frac{\alpha^{2}}{n}\right)\left(\sum_{r \geq 0} \frac{1}{r !} \frac{\alpha^{4 r}}{n^{3 r}}\left[\sum_{t \geq 0} \frac{-1}{(t+2)(2 t+3)} \frac{\alpha^{2 t}}{n^{2 t}}\right]^{r}\right) .
\end{aligned}
$$

We also use

$$
\frac{1}{n \pm \alpha}=\frac{1}{n} \frac{1}{1 \pm \frac{\alpha}{n}}=\frac{1}{n} \sum_{r \geq 0}\left(\mp \frac{\alpha}{n}\right)^{r}
$$

By the symmetry of the binomial coefficient, the resulting asymptotic expansion has to be symmetric in $\alpha$. Assembling all these expansions yields the asymptotic formula

$$
\left(\begin{array}{c}
2 n \\
n-\alpha
\end{array}\right) \sim \frac{4^{n}}{\sqrt{n \pi}} \exp \left(-\frac{\alpha^{2}}{n}\right) \cdot S(\alpha, n)
$$

where $S(\alpha, n)$ is defined as in the statement of the lemma.

Note that $d_{0}=1$, and thus the first summand of the series in (3.1) is 1 - which gives $c_{00}=1$. Summands where the exponent of $\alpha$ exceeds the exponent of $1 / n$ only occur in the last series, with the maximal difference being induced by $\alpha^{4 r} / n^{3 r}$. Thus, if $j>\frac{2}{3} \ell$, we have $c_{\ell j}=0$. Together with $|\alpha| \leq n^{2 / 3}$, this implies the estimate for $S(\alpha, n)$.

For $|\alpha|>n^{2 / 3}$, we can use the monotonicity of the binomial coefficient to obtain

$$
\left(\begin{array}{c}
2 n \\
n-\alpha
\end{array}\right) \leq\left(\begin{array}{c}
2 n \\
n-\left\lceil n^{2 / 3}\right\rceil
\end{array}\right)
$$

for which the exponential factor ensures fast decay,

$$
\exp \left(-\frac{\left\lceil n^{2 / 3}\right\rceil^{2}}{n}\right)=O\left(\exp \left(-n^{1 / 3}\right)\right)
$$

and as everything else is of polynomial growth, the statement of the lemma follows.

Now that we have an asymptotic expansion for the shifted central binomial coefficient, let us look at our explicit formula in (2.3) again: we have

$$
p_{2 m-1}^{(h)}=\frac{4}{4^{m}} \sum_{k \geq 0}(-1)^{k} \frac{\tau_{h, k}}{m}\left(\begin{array}{c}
2 m \\
m-\tau_{h, k}
\end{array}\right) \cdot \llbracket h+1 \equiv 2 m \bmod 2 \rrbracket,
$$


where $\tau_{h, k}=(h+1)(2 k+1) / 2$. Therefore, the total probability for a random walk of length $2 m-1$ on $\mathbb{N}_{0}$ to be admissible is given by

$$
p_{2 m-1}=\sum_{h \geq 0} p_{2 m-1}^{(h)}=\frac{4}{4^{m}} \sum_{\substack{h, k \geq 0 \\
h+1 \equiv 2 m \bmod 2}}(-1)^{k} \frac{\tau_{h, k}}{m}\left(\begin{array}{c}
2 m \\
m-\tau_{h, k}
\end{array}\right) .
$$

The terms where $\tau_{h, k}>m^{2 / 3}$ can be neglected in view of the last statement in Lemma 3.1, as their total contribution decays faster than any power of $m$ : note that there are only $O\left(m^{2}\right)$ such terms (trivially, $h, k \leq m$ ), each of which contributes $O\left(m \exp \left(-m^{1 / 3}\right)\right)$ to the sum. For all other values of $h$ and $k$, we can replace the binomial coefficient by its asymptotic expansion. This gives us, for any $L>0$,

$$
\begin{aligned}
p_{2 m-1}= & \frac{4}{\sqrt{m \pi}} \sum_{\substack{h, k \geq 0, \tau_{h, k} \leq m^{2 / 3} \\
h+1 \equiv 2 m \bmod 2}}(-1)^{k} \frac{\tau_{h, k}}{m} \exp \left(-\frac{\tau_{h, k}^{2}}{m}\right) \sum_{\ell=0}^{L-1} \sum_{j \geq 0} c_{\ell j} \frac{\tau_{h, k}^{2 j}}{m^{\ell}} \\
& +O\left(\frac{1}{\sqrt{m}} \sum_{\substack{h, k \geq 0, \tau_{h, k} \leq m^{2 / 3} \\
h+1 \equiv 2 m \bmod 2}} \frac{\tau_{h, k}^{2 J(L)+1}}{m^{L+1}}\right)
\end{aligned}
$$

where $J(L) \leq \frac{2}{3} L$ since $c_{\ell j}=0$ for $j>\frac{2}{3} \ell$. Since the sum clearly contains $O\left(\mathrm{~m}^{4 / 3}\right)$ terms, the error is at most $O\left(m^{-1 / 2+4 / 3+2 / 3(2 J(L)+1)-(L+1)}\right)=O\left(m^{1 / 2-L / 9}\right)$. The exponent can be made arbitrarily small by choosing $L$ accordingly. Finally, if we extend the sum to the full range (all integers $h, k \geq 0$ such that $h+1 \equiv 2 m \bmod 2$ ) again, we only get another error term of order $O\left(\exp \left(-m^{1 / 3}\right)\right)$, which can be neglected. In summary, we have

$$
p_{2 m-1} \sim \frac{4}{\sqrt{m \pi}} \sum_{\substack{h, k \geq 0 \\ h+1 \equiv 2 m \bmod 2}}(-1)^{k} \frac{\tau_{h, k}}{m} \exp \left(-\frac{\tau_{h, k}^{2}}{m}\right) \sum_{\ell, j \geq 0} c_{\ell j} \frac{\tau_{h, k}^{2 j}}{m^{\ell}} .
$$

This sum can be analyzed with the help of the Mellin transform and the converse mapping theorem (cf. [6]). In order to follow this approach, we will investigate those terms in (3.4) whose growth is not obvious more precisely. That is, we will focus on the contribution of terms of the form

$$
\sum_{\substack{h, k \geq 0 \\ h+1 \equiv 2 m \bmod 2}}(-1)^{k} \tau_{h, k}^{2 j+1} \exp \left(-\frac{\tau_{h, k}^{2}}{m}\right) .
$$

We are also interested in the expected height and the corresponding variance and higher moments of admissible random walks. Asymptotic expansions for these can be obtained by analyzing moments of the random variable $H_{n}$ with $\mathbb{P}\left(H_{n}=h\right):=\frac{p_{n}^{(h)}}{p_{n}}$, as stated in the introduction. For the sake of convenience, let us consider the $r$-th shifted moment $\mathbb{E}\left(H_{2 m-1}+1\right)^{r}$. We know

$$
\mathbb{E}\left(H_{2 m-1}+1\right)^{r}=\sum_{h \geq 0}(h+1)^{r} \mathbb{P}\left(H_{2 m-1}=h\right)=\frac{\sum_{h \geq 0}(h+1)^{r} p_{2 m-1}^{(h)}}{p_{2 m-1}} .
$$


The asymptotic behavior of the denominator is related to the behavior of the sum from above - and fortunately, the behavior of the numerator is related to the behavior of the very similar sum

$$
\sum_{\substack{h, k \geq 0 \\ h+1 \equiv 2 m \bmod 2}}(-1)^{k} \tau_{h, k}^{2 j+1}(h+1)^{r} \exp \left(-\frac{\tau_{h, k}^{2}}{m}\right) .
$$

The following lemma analyzes sums of this structure asymptotically.

Lemma 3.2. Let $j, r \in \mathbb{N}_{0}$. Then we have

$$
\begin{aligned}
& \sum_{\substack{h, k \geq 0 \\
h+1 \equiv 2 m \bmod 2}}(-1)^{k} \tau_{h, k}^{2 j+1}(h+1)^{r} \exp \left(-\frac{\tau_{h, k}^{2}}{m}\right) \\
= & 2^{r-1} \Gamma\left(j+1+\frac{r}{2}\right) \beta(r+1) m^{j+1+r / 2}+O\left(m^{-K}\right),
\end{aligned}
$$

for any fixed $K>0$, where $\beta(\cdot)$ denotes the Dirichlet beta function.

Remark 3.3. The Dirichlet beta function is also often called Catalan beta function, and it is defined by

$$
\beta(s)=\sum_{k=0}^{\infty} \frac{(-1)^{k}}{(2 k+1)^{s}}
$$

It can be expressed in terms of the Hurwitz zeta function as

$$
\beta(s)=4^{-s}(\zeta(s, 1 / 4)-\zeta(s, 3 / 4)) .
$$

Amongst many other interesting properties, it satisfies the zeta-like functional equation (cf. [12, 3:5:2])

$$
\beta(1-s)=(\pi / 2)^{-s} \sin (\pi s / 2) \Gamma(s) \beta(s),
$$

which also implies that $\beta(s)$ has zeros at all negative odd integers.

Proof of Lemma 3.2. If we substitute $m=x^{-2}$, the left-hand side of (3.5) becomes

$$
f(x):=\sum_{\substack{h, k \geq 0 \\ h+1 \equiv 2 m \bmod 2}}(-1)^{k} \tau_{h, k}^{2 j+1}(h+1)^{r} \exp \left(-\tau_{h, k}^{2} x^{2}\right) .
$$

This is a typical example of a harmonic sum, cf. [6, §3], and the Mellin transform can be applied to obtain its asymptotic behaviour. First of all, it is well known that the Mellin transform of a harmonic sum of the form $f(x)=\sum_{k \geq 1} a_{k} g\left(b_{k} x\right)$ can be factored as $\sum_{k \geq 1} a_{k} b_{k}^{-s} g^{*}(s)$ [6, Lemma 2], provided that the half-plane of absolute convergence of the Dirichlet series $\Lambda(s)=\sum_{k \geq 1} a_{k} b_{k}^{-s}$ has non-empty intersection with the fundamental strip of the Mellin transform $g^{*}$ of the base function $g$. In this particular case, the Dirichlet series is

$$
\Lambda(s):=\sum_{\substack{h, k \geq 0 \\ h+1 \equiv 2 m \bmod 2}}(-1)^{k} \tau_{h, k}^{2 j+1-s}(h+1)^{r},
$$


and the base function is $g(x)=\exp \left(-x^{2}\right)$, with Mellin transform $g^{*}(s)=\frac{1}{2} \Gamma\left(\frac{s}{2}\right)$ and fundamental strip $\langle 0, \infty\rangle$.

Now we simplify the Dirichlet series. For $s \in \mathbb{C}$ with $\mathfrak{R}(s)>2 j+2+r$, the sum

$$
\Lambda(s)=2^{s-(2 j+1)} \sum_{\substack{h, k \geq 0 \\ h+1 \equiv 2 m \bmod 2}}(-1)^{k}(h+1)^{2 j+1+r-s}(2 k+1)^{2 j+1-s}
$$

converges absolutely because it is dominated by the zeta function. In view of the definition of the $\beta$ function, this simplifies to

$$
\Lambda(s)=2^{s-(2 j+1)} \beta(s-(2 j+1)) \kappa_{2 m}(s-(2 j+1+r)),
$$

where $\kappa_{2 m}(s)$ depends on the parity of $2 m$. We find

$$
\kappa_{2 m}(s)=\sum_{\substack{h \geq 0 \\ h+1 \equiv 2 m \bmod 2}}(h+1)^{-s}= \begin{cases}2^{-s} \zeta(s), & \text { for } m \in \mathbb{N} \\ \left(1-2^{-s}\right) \zeta(s), & \text { for } m \notin \mathbb{N}\end{cases}
$$

Thus, the Mellin transform of $f$ is

$$
f^{*}(s)=\Lambda(s) g^{*}(s)=\frac{1}{2} \Gamma\left(\frac{s}{2}\right) 2^{s-(2 j+1)} \beta(s-(2 j+1)) \kappa_{2 m}(s-(2 j+1+r)) .
$$

By the converse mapping theorem (see [6, Theorem 4]), the asymptotic growth of $f(x)$ for $x \rightarrow 0$ can be found by considering the analytic continuation of $f^{*}(s)$ further to the left of the complex plane and investigating its poles. The theorem may be applied because $\Lambda(s)$ has polynomial growth and $\Gamma(s / 2)$ decays exponentially along vertical lines of the complex plane.

We find that $f^{*}(s)$ has a simple pole at $s=2 j+2+r$, which comes from the zeta function in the definition of $\kappa_{2 m}$. There are no other poles: $\beta$ is an entire function, and the poles of $\Gamma$ cancel against the zeros of $\beta$ (at all odd negative integers, see the earlier remark).

The asymptotic contribution from the pole of $f^{*}$ is

$$
\begin{aligned}
\operatorname{Res}\left(f^{*}, s=2 j+2+r\right) \cdot x^{-(2 j+2+r)} & =\frac{1}{2} \Gamma\left(j+1+\frac{r}{2}\right) 2^{r+1} \beta(r+1) \frac{1}{2} x^{-(2 j+2+r)} \\
& =2^{r-1} \Gamma\left(j+1+\frac{r}{2}\right) \beta(r+1) m^{j+1+r / 2}
\end{aligned}
$$

which does not depend on the parity of $2 m$, as the respective residue of $\kappa_{2 m}$ is $\frac{1}{2}$ in either case. Finally, the $O$-term in (3.5) comes from the fact that $f^{*}$ may be continued analytically arbitrarily far to the left in the complex plane without encountering any additional poles.

Remark 3.4. In Lemma 3.2, particular values of the Dirichlet beta function are required. To compute the asymptotic expansions for the first moments, we need $\beta(1)=$ $\pi / 4, \beta(2)=G \approx 0.91597$, as well as $\beta(3)=\pi^{3} / 32$, where $G$ is the Catalan constant. These values are taken from [12, Table 3.7.1]. 
At this point, all that remains to obtain asymptotic expansions is to multiply the contributions resulting from Lemma 3.2 with the correct coefficients and contributions from (3.4).

Theorem 3.5. (Asymptotic analysis of admissible random walks on $\mathbb{N}_{0}$ ) The probability that a random walk on $\mathbb{N}_{0}$ is admissible can be expressed asymptotically as

$$
p_{n}=\sqrt{\frac{\pi}{2 n}}-\frac{5 \sqrt{2 \pi}}{24 \sqrt{n^{3}}}+\frac{127 \sqrt{2 \pi}}{960 \sqrt{n^{5}}}-\frac{1571 \sqrt{2 \pi}}{16128 \sqrt{n^{7}}}-\frac{1896913 \sqrt{2 \pi}}{184320 \sqrt{n^{9}}}+O\left(\frac{1}{\sqrt{n^{11}}}\right)
$$

where $\sqrt{\pi / 2} \approx 1.25331$. The expected height of admissible random walks is given by

$$
\mathbb{E} H_{n}=2 G \sqrt{\frac{2 n}{\pi}}-1+\frac{5 \sqrt{2} G}{6 \sqrt{\pi n}}-\frac{131 \sqrt{2} G}{720 \sqrt{\pi n^{3}}}+\frac{1129 \sqrt{2} G}{12096 \sqrt{\pi n^{5}}}+O\left(\frac{1}{\sqrt{n^{7}}}\right),
$$

where $2 G \sqrt{2 / \pi} \approx 1.46167$, and the variance of $H_{n}$ can be expressed as

$$
\mathbb{V} H_{n}=\frac{\pi^{3}-32 G^{2}}{4 \pi} n+\frac{\pi^{3}-40 G^{2}}{6 \pi}-\frac{\pi^{3}-12 G^{2}}{180 \pi n}+\frac{11 \pi^{3}-265 G^{2}}{1890 \pi n^{2}}+O\left(\frac{1}{n^{3}}\right)
$$

where $\left(\pi^{3}-32 G^{2}\right) /(4 \pi) \approx 0.33092$. Generally, the $r$-th moment is asymptotically given by

$$
\mathbb{E} H_{n}^{r} \sim \frac{2^{r / 2+2}}{\pi} \Gamma\left(\frac{r}{2}+1\right) \beta(r+1) n^{r / 2} .
$$

Moreover, if $\eta=h / \sqrt{n}$ satisfies $3 / \sqrt{\log n}<\eta<\sqrt{\log n} / 2$ and $h \equiv n \bmod 2$, we have the local limit theorem

$$
\begin{aligned}
\mathbb{P}\left(H_{n}=h\right) & =\frac{p_{n}^{(h)}}{p_{n}} \sim \frac{2 \phi(\eta)}{\sqrt{n}} \\
& =\frac{8 \eta}{\pi \sqrt{n}} \sum_{k \geq 0}(-1)^{k}(2 k+1) \exp \left(-\frac{(2 k+1)^{2} \eta^{2}}{2}\right) \\
& =\frac{2 \sqrt{2 \pi}}{\eta^{2} \sqrt{n}} \sum_{k \geq 0}(-1)^{k}(2 k+1) \exp \left(-\frac{\pi^{2}(2 k+1)^{2}}{8 \eta^{2}}\right) .
\end{aligned}
$$

Remark 3.6. The fact that the two series in (3.10) and (3.11) that represent the density $\phi(\eta)$ are equal is a simple consequence of the Poisson sum formula. We also note that the asymptotic behavior of the moments of $H_{n}$ readily implies that the normalized random variable $H_{n} / \sqrt{n}$ converges weakly to the distribution whose density is given by $\phi(\eta)$ (see [7, Theorem C.2]). The local limit theorem (3.10) is somewhat stronger.

Proof of Theorem 3.5. With (3.4) and the result of Lemma 3.2, obtaining an asymptotic expansion of $p_{2 m-1}$ is only a question of developing the shifted central binomial coefficient and multiplying with the correct growth contributions from (3.5). By doing so (with the help of SageMath [15]: a worksheet containing these computations 
as well as some numerical comparisons can be found at http://arxiv.org/src/ 1503.08790/anc/random-walk_NN. ipynb), an asymptotic expansion in the halfinteger $m$ is obtained. Substituting $m=(n+1) / 2$ then gives (3.6).

The results in (3.7) and (3.8) are obtained by considering

$$
\mathbb{E}\left(H_{n}+1\right)^{r}=\frac{\sum_{h \geq 0}(h+1)^{r} p_{n}^{(h)}}{p_{n}},
$$

making use of (3.4) and Lemma 3.2 again. Note that we have $\mathbb{E} H_{n}=\mathbb{E}\left(H_{n}+1\right)-1$, as well as $\mathbb{V} H_{n}=\mathbb{E}\left(H_{n}+1\right)^{2}-\left[\mathbb{E}\left(H_{n}+1\right)\right]^{2}$. For higher moments, we only give the principal term of the asymptotics, which corresponds to the coefficient $c_{00}$ in (3.4), but in principle it would be possible to calculate further terms as well.

It remains to prove (3.10). To this end, we revisit the explicit expression (recall that we set $n=2 m-1$ )

$$
p_{2 m-1}^{(h)}=\frac{4}{4^{m}} \sum_{k \geq 0}(-1)^{k} \frac{\tau_{h, k}}{m}\left(\begin{array}{c}
2 m \\
m-\tau_{h, k}
\end{array}\right) .
$$

First of all, we can eliminate all $k$ with $\tau_{h, k}>m^{2 / 3}$, since their total contribution is at most $O\left(m \exp \left(-m^{1 / 3}\right)\right)$ as before. For all other values of $k$, we replace the binomial coefficient according to Lemma 3.1 by

$$
\left(\begin{array}{c}
2 m \\
m-\tau_{h, k}
\end{array}\right)=\frac{4^{m}}{\sqrt{\pi m}} \exp \left(-\frac{\tau_{h, k}^{2}}{m}\right)\left(1+O\left(\frac{1+\tau_{h, k}}{m}\right)\right) .
$$

Note here that

$$
\tau_{h, k}=\frac{(h+1)(2 k+1)}{2}=\frac{h}{2}(2 k+1)\left(1+O\left(\frac{1}{h}\right)\right),
$$

and likewise,

$$
\frac{\tau_{h, k}^{2}}{m}=\frac{h^{2}(2 k+1)^{2}}{2 n}\left(1+O\left(\frac{1}{h}+\frac{1}{n}\right)\right) .
$$

It follows that

$$
\frac{\tau_{h, k}}{m} \exp \left(-\frac{\tau_{h, k}^{2}}{m}\right)=\frac{h(2 k+1)}{n} \exp \left(-\frac{h^{2}(2 k+1)^{2}}{2 n}\right)\left(1+O\left(\frac{1}{h}+\frac{h k^{2}+1}{n}\right)\right) .
$$

We are assuming that $\tau_{h, k} \leq m^{2 / 3}=((n+1) / 2)^{2 / 3}$, which implies $h k^{2} / n=O\left(n^{1 / 3} / h\right)$. In view of our assumptions on $h$, this means that the error term is $O\left(n^{-1 / 6} \sqrt{\log n}\right)$. Thus we have

$$
\begin{aligned}
p_{n}^{(h)}= & p_{2 m-1}^{(h)} \\
= & \frac{4 \sqrt{2} h}{\sqrt{\pi n^{3}}} \times \sum_{\substack{k \geq 0 \\
\tau_{h, k} \leq((n+1) / 2)^{2 / 3}}}(-1)^{k}(2 k+1) \exp \left(-\frac{h^{2}(2 k+1)^{2}}{2 n}\right)\left(1+O\left(\frac{\sqrt{\log n}}{n^{1 / 6}}\right)\right) \\
& +O\left(n \exp \left(-(n / 2)^{1 / 3}\right)\right) .
\end{aligned}
$$


Adding all terms $\tau_{h, k}>m^{2 / 3}=((n+1) / 2)^{2 / 3}$ back only results in a negligible contribution that decays faster than any power of $n$ again, but we need to be careful with the $O$-term inside the sum, as we have to bound the accumulated error by the sum of the absolute values. We have

$$
\sum_{k \geq 0}(2 k+1) \exp \left(-\frac{h^{2}(2 k+1)^{2}}{2 n}\right)=O\left(n / h^{2}\right),
$$

which can be seen, e.g., by approximating the sum by an integral (or by means of the Mellin transform again), so

$$
\begin{aligned}
p_{n}^{(h)} & =\frac{4 \sqrt{2} h}{\sqrt{\pi n^{3}}} \sum_{k \geq 0}(-1)^{k}(2 k+1) \exp \left(-\frac{h^{2}(2 k+1)^{2}}{2 n}\right)+O\left(\frac{\sqrt{\log n}}{h n^{2 / 3}}\right) \\
& =\frac{4 \sqrt{2} \eta}{\sqrt{\pi} n} \sum_{k \geq 0}(-1)^{k}(2 k+1) \exp \left(-\frac{h^{2}(2 k+1)^{2}}{2 n}\right)+O\left(\frac{\log n}{n^{7 / 6}}\right) .
\end{aligned}
$$

Since $p_{n}=\sqrt{\frac{\pi}{2 n}}\left(1+O\left(n^{-1}\right)\right)$, this yields

$$
\begin{aligned}
\frac{p_{n}^{(h)}}{p_{n}} & =\frac{8 \eta}{\pi \sqrt{n}} \sum_{k \geq 0}(-1)^{k}(2 k+1) \exp \left(-\frac{\eta^{2}(2 k+1)^{2}}{2}\right)+O\left(\frac{\log n}{n^{2 / 3}}\right) \\
& =\frac{2 \phi(\eta)}{\sqrt{n}}+O\left(\frac{\log n}{n^{2 / 3}}\right) .
\end{aligned}
$$

For $\eta \geq 1$, the sum is bounded below by a constant multiple of $\exp \left(-\eta^{2} / 2\right)$ (as can be seen by bounding the sum of all terms with $k \geq 1$ ), which in turn is at least $\exp (-(\log n) / 8)=n^{-1 / 8}$ by our assumptions on $\eta$. Thus the first term indeed dominates the error term in this case. If $\eta<1$, we use the alternative representation (3.11), which shows that $\phi(\eta)$ is bounded below by a constant multiple of $\eta^{-2} \exp \left(-\pi^{2} /(8 \eta)^{2}\right)$. This in turn is at least $(1 / 9) n^{-\pi^{2} / 72} \log n$ by the assumptions on $\eta$, and since $\pi^{2} / 72<1 / 6$, we can draw the same conclusion.

Remark 3.7. As stated in the introduction, the number $2^{n} p_{n}$ gives the number of extremal lattice paths on $\mathbb{Z}$ - and thus, with the asymptotic expansion of $p_{n}$, we also have an asymptotic expansion for the number of extremal lattice paths on $\mathbb{Z}$ of given length.

This concludes our analysis of admissible random walks on $\mathbb{N}_{0}$. In the next section, we investigate admissible random walks on $\mathbb{Z}$.

\section{Ballot Sequences and Admissible Random Walks on $\mathbb{Z}$}

In principle, the approach we follow for the analysis of the asymptotic behavior of admissible random walks on $\mathbb{Z}$ is the same as in the previous section. However, due to the different structure of (2.4), some steps will need to be adapted. 
With the notation of Lemma 3.1, we are able to express $q_{2 m-2}$ for a half-integer $m \in \frac{1}{2} \mathbb{N}$ with $m \geq 1$ as

$$
q_{2 m-2} \sim \frac{4}{\sqrt{m \pi}} \frac{1}{2 m-1} \sum_{\substack{h, k \geq 0 \\ h \equiv 2 m \bmod 2}} \frac{2 v_{h, k}^{2}-m}{m} \exp \left(-\frac{v_{h, k}^{2}}{m}\right) \sum_{\ell, j \geq 0} c_{\ell, j} \frac{v_{h, k}^{2 j}}{m^{\ell}} .
$$

In analogy to our investigation of admissible random walks on $\mathbb{N}_{0}$, we also want to determine the expected height and variance of admissible random walks. These are related to the random variable $\widetilde{H}_{n}$, which we defined by

$$
\mathbb{P}\left(\widetilde{H}_{n}=h\right)=\frac{q_{n}^{(h)}}{q_{n}}
$$

To make things easier, we will investigate moments of the form $\mathbb{E}\left(\widetilde{H}_{n}+2\right)^{r}$. They can be computed by

$$
\mathbb{E}\left(\widetilde{H}_{n}+2\right)^{r}=\sum_{h \geq 0}(h+2)^{r} \mathbb{P}\left(\widetilde{H}_{n}=h\right)=\frac{\sum_{h \geq 0}(h+2)^{r} q_{n}^{(h)}}{q_{n}} .
$$

Therefore, we are interested in the asymptotic contribution of

$$
\sum_{\substack{h, k \geq 0 \\ h \equiv 2 m \bmod 2}} \frac{2 v_{h, k}^{2}-m}{m} v_{h, k}^{2 j}(h+2)^{r} \exp \left(-\frac{v_{h, k}^{2}}{m}\right)
$$

which is discussed in the following lemma.

Lemma 4.1. Let $K>0$ be fixed. Then we have the asymptotic expansion

$$
\sum_{\substack{h, k \geq 0 \\ h \equiv 2 m \bmod 2}} \frac{2 v_{h, k}^{2}-m}{m} \exp \left(-\frac{v_{h, k}^{2}}{m}\right)=\frac{\sqrt{m \pi}}{4}+O\left(m^{-K}\right) .
$$

For $j \in \mathbb{N}$ we have

$$
\begin{aligned}
& \sum_{\substack{h, k \geq 0 \\
h \equiv 2 m \bmod 2}} \frac{2 v_{h, k}^{2}-m}{m} v_{h, k}^{2 j} \exp \left(-\frac{v_{h, k}^{2}}{m}\right) \\
& =\left(\frac{\log m}{2}+2 \gamma+\log 2+\frac{1}{2} \psi\left(j+\frac{1}{2}\right)+\frac{1}{2 j}+\llbracket m \notin \mathbb{N} \rrbracket \cdot(2 \log 2-2)\right) \\
& \quad \times \frac{j}{2} \Gamma\left(j+\frac{1}{2}\right) m^{j+1 / 2}+O\left(m^{-K}\right),
\end{aligned}
$$


where $\psi(s)$ is the digamma function. Finally, for $j \in \mathbb{N}_{0}, r \in \mathbb{N}$ we find

$$
\begin{aligned}
& \sum_{\substack{h, k \geq 0 \\
h \equiv 2 m \bmod 2}} \frac{2 v_{h, k}^{2}-m}{m} v_{h, k}^{2 j}(h+2)^{r} \exp \left(-\frac{v_{h, k}^{2}}{m}\right) \\
= & j \Gamma\left(j+\frac{1}{2}\right) \kappa_{2 m}(1-r) m^{j+1 / 2} \\
& +\frac{1}{2}\left(j+\frac{r}{2}\right) \Gamma\left(j+\frac{r+1}{2}\right)\left(2^{r+1}-1\right) \zeta(r+1) m^{j+(r+1) / 2}+O\left(m^{-K}\right),
\end{aligned}
$$

where $\kappa_{2 m}(s)=2^{-s} \zeta(s)$ for $m \in \mathbb{N}$ and $\kappa_{2 m}(s)=\left(1-2^{-s}\right) \zeta(s)-1$ otherwise.

Proof. Let $j, r \in \mathbb{N}_{0}$. We want to analyze the sum

$$
\sum_{\substack{h, k \geq 0 \\ h \equiv 2 m \bmod 2}} \frac{2 v_{h, k}^{2}-m}{m} v_{h, k}^{2 j}(h+2)^{r} \exp \left(-\frac{v_{h, k}^{2}}{m}\right)
$$

asymptotically, where $m$ is a half-integer in $\frac{1}{2} \mathbb{N}$ with $m \geq 1$.

In analogy to the proof of Lemma 3.2, we substitute $x^{-2}=m$, so that the sum becomes

$$
\begin{aligned}
f(x) & :=\sum_{\substack{h, k \geq 0 \\
h \equiv 2 m \bmod 2}}\left(2 x^{2} v_{h, k}^{2}-1\right) v_{h, k}^{2 j}(h+2)^{r} \exp \left(-v_{h, k}^{2} x^{2}\right) \\
= & 2 x^{2} \sum_{\substack{h, k \geq 0 \\
h \equiv 2 m \bmod 2}} v_{h, k}^{2 j+2}(h+2)^{r} \exp \left(-v_{h, k}^{2} x^{2}\right) \\
& -\sum_{\substack{h, k \geq 0 \\
h \equiv 2 m \bmod 2}} v_{h, k}^{2 j}(h+2)^{r} \exp \left(-v_{h, k}^{2} x^{2}\right) \\
=: & 2 x^{2} f_{1}(x)-f_{2}(x) .
\end{aligned}
$$

Both $f_{1}$ and $f_{2}$ are harmonic sums, and we determine their Mellin transforms as we did earlier in the proof of Lemma 3.2. By elementary properties of the Mellin transform, we know that $f^{*}(s)=2 f_{1}^{*}(s+2)-f_{2}^{*}(s)$. Let $\Lambda_{1}$ and $\Lambda_{2}$ be the Dirichlet series associated with the harmonic sums $f_{1}(x)$ and $f_{2}(x)$, respectively. We find

$$
\begin{aligned}
\Lambda_{1}(s) & =\sum_{\substack{h, k \geq 0 \\
h \equiv 2 m \bmod 2}} v_{h, k}^{2 j+2-s}(h+2)^{r} \\
& =2^{s-(2 j+2)} \sum_{\substack{h, k \geq 0 \\
h \equiv 2 m \bmod 2}}(h+2)^{2 j+2+r-s}(2 k+1)^{2 j+2-s} \\
& =\left(2^{s-(2 j+2)}-1\right) \zeta(s-(2 j+2)) \sum_{\substack{h \geq 0 \\
h \equiv 2 m \bmod 2}}(h+2)^{2 j+2+r-s} .
\end{aligned}
$$


We investigate the sum over $h$ separately, and obtain

$$
\kappa_{2 m}(s):=\sum_{\substack{h \geq 0 \\ h \equiv 2 m \bmod 2}}(h+2)^{-s}= \begin{cases}2^{-s} \zeta(s), & \text { for } m \in \mathbb{N}, \\ \left(1-2^{-s}\right) \zeta(s)-1, & \text { for } m \notin \mathbb{N} .\end{cases}
$$

Therefore, we find the Mellin transform of the first harmonic sum to be

$$
f_{1}^{*}(s)=\frac{1}{2} \Gamma\left(\frac{s}{2}\right) \Lambda_{1}(s)=\frac{1}{2} \Gamma\left(\frac{s}{2}\right)\left(2^{s-2 j-2}-1\right) \zeta(s-2 j-2) \kappa_{2 m}(s-(2 j+r)-2) .
$$

The Mellin transform of the second sum can be found in a completely analogous way: we have

$$
f_{2}^{*}(s)=\frac{1}{2} \Gamma\left(\frac{s}{2}\right)\left(2^{s-2 j}-1\right) \zeta(s-2 j) \kappa_{2 m}(s-(2 j+r)) .
$$

Altogether, this yields the Mellin transform

$$
\begin{aligned}
f^{*}(s) & =2 f_{1}^{*}(s+2)-f_{2}^{*}(s) \\
& =\frac{s-1}{2} \Gamma\left(\frac{s}{2}\right)\left(2^{s-2 j}-1\right) \zeta(s-2 j) \kappa_{2 m}(s-(2 j+r)) .
\end{aligned}
$$

As in the proof of Lemma 3.2, the growth conditions necessary for application of the converse mapping theorem [6, Theorem 4] hold.

In order to analyze the poles of $f^{*}(s)$, we need to distinguish three cases, as $\zeta(s-2 j)$ has a simple pole at $s=2 j+1$ and $\kappa_{2 m}(s-(2 j+r))$ has a simple pole at $s=2 j+r+1$. The poles of $\Gamma(s / 2)$ at even $s \leq 0$ are canceled by the zeros of $\zeta(s-2 j)$, unless $s=j=0$. In that case, the pole is canceled by the factor $\left(2^{s-2 j}-1\right)$.

First, let $r=j=0$. Then, $f^{*}(s)$ has a simple pole at $s=1$, because one of the poles of $\zeta(s)$ or $\kappa_{2 m}(s)$ cancels against the zero of $(s-1)$. Here, the residue of $f^{*}(s)$ is given by $\sqrt{\pi} / 4$, which translates to a contribution of $\sqrt{m \pi} / 4$. This proves (4.2).

Second, for $r=0$ and $j>0$, the function $f^{*}(s)$ has a pole of order 2 at $s=2 j+1$. By expanding all the occurring functions, we find the Laurent expansion

$f^{*}(s) \asymp \begin{cases}\frac{j}{2} \Gamma\left(j+\frac{1}{2}\right)\left[\frac{1}{(s-(2 j+1))^{2}}+\frac{\frac{1}{2} \psi\left(j+\frac{1}{2}\right)+2 \gamma+\log 2+\frac{1}{2 j}}{s-(2 j+1)}\right]+O(1), & \text { for } m \in \mathbb{N}, \\ \frac{j}{2} \Gamma\left(j+\frac{1}{2}\right)\left[\frac{1}{(s-(2 j+1))^{2}}+\frac{\frac{1}{2} \psi\left(j+\frac{1}{2}\right)+2 \gamma+3 \log 2-2+\frac{1}{2 j}}{s-(2 j+1)}\right]+O(1), & \text { for } m \notin \mathbb{N},\end{cases}$

where $\psi(s)$ is the digamma function (cf. [5, 5.2.2], see [5, $\S 5.4$ (ii)] for special values). As the pole of order 2 contributes the factor $\frac{1}{2} m^{j+1 / 2} \log m$, and the pole of order 1 gives $m^{j+1 / 2},(4.3)$ is proved.

Finally, consider $r>0$. In this case we have two separate single poles at $s=2 j+1$ and $s=2 j+r+1$. Computing the residues gives the growth contribution

$$
\begin{aligned}
& j \Gamma\left(j+\frac{1}{2}\right) \kappa_{2 m}(1-r) m^{j+1 / 2} \\
& +\left(j+\frac{r}{2}\right) \Gamma\left(j+\frac{r+1}{2}\right)\left(2^{r}-\frac{1}{2}\right) \zeta(r+1) m^{j+(r+1) / 2},
\end{aligned}
$$

which proves (4.4). 
Fortunately, when explicitly computing the expansion, all the logarithmic terms cancel out and we obtain the same behavior for admissible paths of even and odd length. The following theorem summarizes our findings.

Theorem 4.2. (Asymptotic analysis of admissible random walks on $\mathbb{Z}$ ) The probability that a random walk on $\mathbb{Z}$ is admissible has the asymptotic expansion

$$
q_{n}=\frac{1}{n}-\frac{4}{3 n^{2}}+\frac{88}{45 n^{3}}-\frac{976}{315 n^{4}}+\frac{3488}{675 n^{5}}-\frac{276928}{31185 n^{6}}+O\left(\frac{1}{n^{7}}\right) .
$$

The expected height of admissible random walks on $\mathbb{Z}$ is given by

$$
\mathbb{E} \widetilde{H}_{n}=\frac{\sqrt{2 \pi^{3}}}{4} \sqrt{n}-2+\frac{3 \sqrt{2 \pi^{3}}}{16 \sqrt{n}}-\frac{539 \sqrt{2 \pi^{3}}}{5760 \sqrt{n^{3}}}+\frac{50713 \sqrt{2 \pi^{3}}}{483840 \sqrt{n^{5}}}+O\left(\frac{1}{\sqrt{n^{7}}}\right),
$$

where $\sqrt{2 \pi^{3}} / 4 \approx 1.96870$, and the variance of $\widetilde{H}_{n}$ can be expressed as

$$
\begin{aligned}
\mathbb{V} \widetilde{H}_{n}= & \frac{28 \zeta(3)-\pi^{3}}{8} n+\frac{224 \zeta(3)-9 \pi^{3}}{48}-\frac{1792 \zeta(3)-67 \pi^{3}}{2880 n} \\
& +\frac{107520 \zeta(3)-4189 \pi^{3}}{120960 n^{2}}+O\left(\frac{1}{n^{3}}\right)
\end{aligned}
$$

where $\left(28 \zeta(3)-\pi^{3}\right) / 8 \approx 0.33141$. Generally, the $r$-th moment is asymptotically given by

$$
\mathbb{E} \widetilde{H}_{n}^{r} \sim \frac{r}{\sqrt{\pi}} \Gamma\left(\frac{r+1}{2}\right)\left(2^{r+1}-1\right) 2^{-r / 2} \zeta(r+1) n^{r / 2} .
$$

Moreover, if $\eta=h / \sqrt{n}$ satisfies $6 / \sqrt{\log n}<\eta<\sqrt{\log n} / 2$, we have the local limit theorem

$$
\begin{aligned}
\mathbb{P}\left(\widetilde{H}_{n}=h\right)=\frac{q_{n}^{(h)}}{q_{n}} \sim \frac{2 \chi(\eta)}{\sqrt{n}} & =\frac{4 \sqrt{2}}{\sqrt{\pi n}} \sum_{k \geq 0}\left((2 k+1)^{2} \eta^{2}-1\right) \exp \left(-\frac{(2 k+1)^{2} \eta^{2}}{2}\right) \\
& =\frac{4 \pi^{2}}{\eta^{3} \sqrt{n}} \sum_{k \geq 1}(-1)^{k-1} k^{2} \exp \left(-\frac{\pi^{2} k^{2}}{2 \eta^{2}}\right) .
\end{aligned}
$$

Remark 4.3. Again, the two expressions for the limiting density $\chi$ are equivalent, as can be seen by an application of the Poisson sum formula.

Proof of Theorem 4.2. Analogous to Theorem 3.5. The asymptotic expansions were again computed with the help of SageMath [15], and a corresponding worksheet (containing these computations as well as some numerical comparisons) can be found at http://arxiv.org/src/1503.08790/anc/random-walk_ZZ.ipynb.

Remark 4.4. As every simple symmetric random walk of length $n$ on $\mathbb{Z}$ occurs with probability $2^{-n}$, we know that the number of admissible random walks on $\mathbb{Z}$ is $2^{n} q_{n}$. Thus, an asymptotic expansion for the number of admissible random walks follows directly from (4.5) upon multiplication by $2^{n}$. This is sequence A167510 in [11]. 


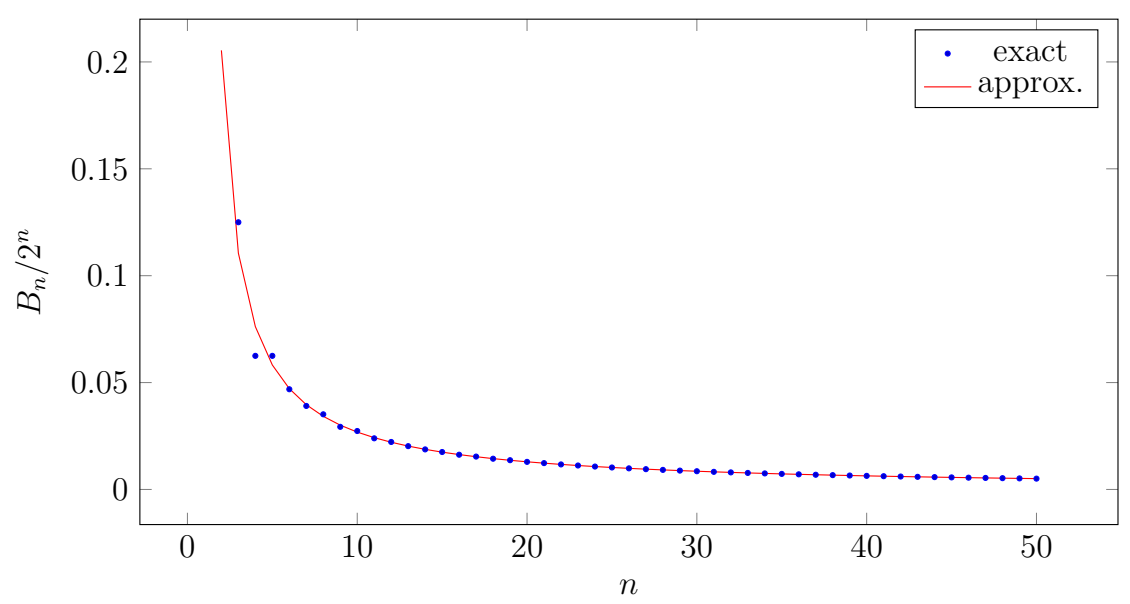

Figure 3: Numerical approximation of $B_{n} / 2^{n}$.

Furthermore, in the introduction we illustrated that admissible random walks are strongly related to bidirectional ballot sequences. Since every bidirectional ballot sequence of length $n+2$ corresponds to an admissible random walk of length $n$ on $\mathbb{Z}$ (i.e., $B_{n}=2^{n-2} q_{n-2}$ ), we are able to prove Zhao's conjecture that was mentioned in the introduction.

Corollary 4.5. (Bidirectional ballot walks) The number of bidirectional ballot walks $B_{n}$ of length $n$ can be expressed asymptotically as

$$
B_{n}=2^{n}\left(\frac{1}{4 n}+\frac{1}{6 n^{2}}+\frac{7}{45 n^{3}}+\frac{10}{63 n^{4}}+\frac{764}{4725 n^{5}}+\frac{4952}{31185 n^{6}}\right)+O\left(\frac{2^{n}}{n^{7}}\right) .
$$

In Figure 3 we compare the exact values of $B_{n} / 2^{n}$ with the values obtained from the asymptotic expansion in (4.11).

\section{References}

1. Aigner, M.: A Course in Enumeration. Graduate Texts in Mathematics, Vol. 238. Springer, Berlin (2007)

2. Banderier, C., Flajolet, P.: Basic analytic combinatorics of directed lattice paths. Theoret. Comput. Sci. 281(1-2), 37-80 (2002)

3. Bousquet-Mélou, M., Ponty, Y.: Culminating paths. Discrete Math. Theor. Comput. Sci. 10(2), 125-152 (2008)

4. de Bruijn, N.G., Knuth, D.E., Rice, S.O.: The average height of planted plane trees. In: Read, R.C. (ed.) Graph Theory and Computing, pp. 15-22. Academic Press, New York (1972)

5. NIST Digital library of mathematical functions, http://dlmf.nist.gov/, Release 1.0.9 of 2014-08-29, Online companion to [13] (2010)

6. Flajolet, P., Gourdon, X., Dumas, P.: Mellin transforms and asymptotics: harmonic sums. Theoret. Comput. Sci. 144(1-2), 3-58 (1995) 
7. Flajolet, P., Sedgewick, R.: Analytic Combinatorics. Cambridge University Press, Cambridge (2009)

8. Graham, R.L., Knuth, D.E., Patashnik, O.: Concrete Mathematics: a Foundation for Computer Science. Second edition. Addison-Wesley, Reading, MA (1994)

9. Jiu, L., Moll, V.H., Vignat, C.: Identities for generalized Euler polynomials. Integral Transforms Spec. Funct. 25(10), 777-789 (2014)

10. Nemes, G.: On the coefficients of the asymptotic expansion of $n$ !. J. Integer Seq. 13(6), Art. 10.6.6 (2010)

11. The On-Line Encyclopedia of Integer Sequences, http://oeis . org (2015)

12. Oldham, K., Spanier, J.: An Atlas of Functions. Taylor \& Francis/Hemisphere, Bristol, PA (1987)

13. Olver, F.W.J., Lozier, D.W., Boisvert, R.F., Clark, C.W.: NIST Handbook of Mathematical Functions. Cambridge University Press, New York (2014)

14. Panny, W., Prodinger, H.: The expected height of paths for several notions of height. Studia Sci. Math. Hungar. 20(1-4), 119-132 (1985)

15. Stein, W.A. et al.: Sage Mathematics Software (Version 6.5). The Sage Development Team (2015)

16. Zhao, Y.: Constructing MSTD sets using bidirectional ballot sequences. J. Number Theory 130(5), 1212-1220 (2010)

Acknowledgments Open access funding provided by [Austrian university].

Open Access This article is distributed under the terms of the Creative Commons Attribution 4.0 International License (http://creativecommons.org/licenses/by/4.0/), which permits unrestricted use, distribution, and reproduction in any medium, provided you give appropriate credit to the original author(s) and the source, provide a link to the Creative Commons license, and indicate if changes were made. 